\title{
SIMULTANEOUS CELL TRACKING AND IMAGE ALIGNMENT IN 3D CLSM IMAGERY OF GROWING ARABIDOPSIS THALIANA SEPALS
}

\author{
R.H.J. Fick \\ D. Fedorov ${ }^{\star \star}$ \\ A.H.K. Roeder ${ }^{\star \star \star}$ \\ B.S. Manjunath ${ }^{\star \star}$ \\ * Department of Biomedical Image Analysis, Eindhoven University of Technology, The Netherlands \\ ${ }^{\star \star}$ Center for Bio-Image Informatics, University of California, Santa Barbara, CA \\ ${ }^{\star \star \star}$ Department of Plant Biology and Weill Institute, Cornell University, Ithaca, NY
}

\begin{abstract}
In this research we propose a combined cell matching and image alignment method for tracking cells based on their nuclear locations in 3D fluorescent Confocal Laser Scanning Microscopy (CLSM) image sequences. We then apply it to study the cell division pattern in the developing sepal of the small plant Arabidopsis thaliana. The method is based on geometric hashing and inherits its invariance to rotation, translation and scale. The method consists of three steps. In the first step the centroids of nuclei are detected using a previously developed cell detection algorithm, reducing the CLSM volumes to $3 \mathrm{D}$ point clouds, wherein every point represents a nuclear centroid with an associated confidence level. In the second step centroids between images are matched in two phases. First geometric hashing is used to find an initial set of centroid matches, then using the initial matches a dense matching is obtained through a novel iterative point matching algorithm. In the last step centroid matches are used to estimate transformations and register all input images to a common frame. Our algorithm has successfully aligned 12 volumes encompassing 72 hours data set and matched 258 nuclear lifelines.
\end{abstract}

\section{INTRODUCTION}

To better understand plant development at a cellular level, biologists would need to track development from a single cell to a complex multi-cellular organism. Currently, this is limited to tracking several cells throughout some stages of development. The process of development in Arabidopsis thaliana is of special interest because of its short life cycle. Specifically, the sepal is a useful model system for examining the role of growth and cell division in patterning of the organ because it is accessible for imaging and manipulation. The outer epidermis of the sepal contains a characteristic pattern of giant cells, which stretch a fifth of the length of the sepal, interspersed between smaller cells. This pattern of different cell sizes is tightly intertwined with cell division and growth: it arises from variation in the times when cells stop dividing and enter a specialized endoreduplication cell cycle $[1,2]$.

Recent research focuses on computational modeling of cells and their patterns in the developing plant by simulating the developmental processes under different conditions [1]. Such modeling provides a new way of understanding the complex interaction networks underlying the development and maintenance of a plant. However, in order to validate these models, quantification of real data provided by imaging techniques is essential. In particular, live 3D fluorescent imaging is being used. In 3D fluorescent microscopy the sepal is periodically imaged by positioning the plant under the microscope. Individual cells are then tracked through the 3D fluorescent imaging data set and cell behavior is compared to computational models.

Cell tracking through 4D data sets is currently still often a manual process, in which cells between images are matched one by one [3]. Considering that every image can contain hundreds of cells, this is an extremely time consuming and arduous task prone to errors and inconsistencies.

For this reason we introduce a novel automatic cell matching method based on image analysis. The method uses local geometry to match cells, and does not require a pre-aligned data set, which is required for other cell lineage tracing methods [4]. Automating the cell matching process will allow plant biologists to employ a so called "high throughput biology" methodology for future experiments. This means that a very large amount of experiments could be performed in parallel to obtain information on cell development much faster.

\section{DATA SET DESCRIPTION}

The experimental data set consists of twelve $3 D$ fluorescent microscopy images of a single Arabidopsis thaliana sepal developing over 72 hours. The time between every image is 6 hours. Figure 1 shows the first, middle and last image of the data set. The points projected over the image volumes represent the centroids detected by the nuclei detection algorithm. A confidence of detection from 0 to 1 is assigned to each centroid, visually color-coded from blue to yellow, respectively. As the sepal grows, cells generally move outward and cell divisions take place, meaning that nuclei move non-linearly, and new nuclei appear. The image dimensions, voxel sizes 
and number of detected nuclei for all images also vary between $512 \times 512 \times 48$ to $512 \times 512 \times 94$ voxels, $0.3 \times 0.3 \times 1$ to $0.6 \times 0.6 \times 1$ microns and 175 to 909 centroids, respectively. The image resolution decreases as time progresses to keep the entirety of the growing sepal in view. Frame 8 is missing from the data set, meaning that there is a 12 hour interval between frames 7 and 9 . The number of nuclei does not increase monotonically because parts of other sepals are in the edges of the images, and some nuclei located outside of the area of interest are detected by the detection algorithm.

\section{METHODOLOGY}

At first, the centroids of cells are detected using a previously developed and validated cell detection algorithm [5]. The centroid detection reduces CLSM volumes to 3D point clouds, wherein every point represents a nuclear centroid with an associated confidence level. Centroid positions are then converted from pixels to microns in order to normalize for resolution.

The matching algorithm starts by finding an initial set of matches between two images using geometric hashing. Then an iterative matching algorithm is used to obtain a dense matching. The resulting centroid matches are then used to transform all CLSM volumes to a common frame.

The algorithm was implemented and tested in Mathematica [6] and is being converted to Matlab for execution on the cluster resources.

\subsection{Initial Matching}

To find an initial set of matches a technique called geometric hashing is used [7]. Geometric hashing is a rotation, translation, and scale invariant method for finding an object $A$ in an input data set $B$, both represented by discrete points. The method is adapted for the purpose of matching two centroid images, and consists of a preprocessing phase, wherein the positions of a small group of neighboring centroids are described by a local basis for every centroid in every frame, followed by a recognition phase, wherein the initial matches between every two consecutive frames are identified. In the preprocessing phase for every frame a symmetric $N \times N$ distance matrix $\mathbf{D}$ is created where the $(i, j)$ element $D_{i j}$ represents the Euclidean distance between centroids $c_{i}$ and $c_{j}$, where $i, j=1, \ldots, N$ with $N$ the number of centroids in the frame. Then for every centroid $c_{i}$ a subset of centroids $S_{i}$ is created. A centroid $c_{j}$ belongs to subset $S_{i}$ when the distance between centroids $c_{i}$ and $c_{j}$ is smaller than 4 times an image dependent threshold $d$, where $d$ is the average nearest neighbor distance for all centroids in a frame.

$$
c_{j} \in S_{i} \text { if } D_{i j}<4 d \text { with } d=\frac{1}{N} \sum_{i=1}^{N} \min \left(D_{i}\right)
$$

The threshold of $4 d$ was found to define an appropriately small area without having to explicitly set a threshold in mi- crons. The following 3 steps are then performed for every subset $S_{i}$ : (1) The centroids in $S_{i}$ are sorted by increasing distance to centroid $c_{i}$. (2) Centroid positions $S_{(i, 1)}, S_{(i, 2)}$ and $S_{(i, 3)}$ in the sorted subset are selected as a basis triplet to form a local basis, described by origin $O$ and axes vectors $X$, $Y$ and $Z$ in the following way:

$$
\begin{aligned}
O & =\frac{S_{(i, 1)}+S_{(i, 2)}+S_{(i, 3)}}{3} \\
X & =\frac{S_{(i, 2)}-S_{(i, 1)}}{\left\|S_{(i, 2)}-S_{(i, 1)}\right\|^{2}} \\
Y & =\frac{X \times\left(S_{(i, 3)}-S_{(i, 1)}\right)}{\left\|X \times\left(S_{(i, 3)}-S_{(i, 1)}\right)\right\|^{2}} \\
Z & =X \times Y
\end{aligned}
$$

(3) The centroid positions in the subset are then mapped to the local basis by subtracting the origin and then multiplication with the basis axes vectors

$$
H_{(i, k)}=\left(S_{(i, k)}-O\right) \cdot(X, Y, Z)^{\mathrm{T}} .
$$

where $H_{(i, k)}$ and $S_{(i, k)}$ are the mapped and subset positions, respectively, with $k$ indicating the index of a specific position in the subset. The list of mapped centroids $H_{i}$ is called the hash table. When all hash tables have been formed, the preprocessing phase is complete.

Next is the recognition phase. First, overlapping hash table positions are identified between every frame $A$ and following frame $B$. Hash positions $H_{(i, k)}^{A}$ and $H_{(j, p)}^{B}$ match if the distance between them is smaller than threshold $d / 2$

$$
\left(H_{(i, k)}^{A}, H_{(j, p)}^{B}\right) \text { match if }\left\|H_{(i, k)}^{A}-H_{(j, p)}^{B}\right\|<d / 2
$$

where $i=1, \ldots, N_{A}$ and $j=1, \ldots, N_{B}$ with $N_{A}$ and $N_{B}$ the number of hash tables in frames $A$ and $B$, respectively, and $k$ and $p$ indicate a specific position index. The matching threshold $d / 2$ was found to define an appropriately small matching threshold. After all overlaps have been identified, every hash table in frame $A$ will have a number of overlaps with every hash table in frame $B$. To identify which hash table combinations are correct, all hash table matches are indexed according to their corresponding centroid matches. Correct centroid matches are usually detected by several hash table combinations. Therefore, matches between hash tables with overlapping centroid matches are joined in one group. This will usually result in one larger group which has mostly correct matches and some smaller groups. Initially only one-toone matching is accepted, so when one or more centroids are matched to one or more centroids in a following frame, only the match with the most occurrences in the group is accepted. The matches in the largest resulting group are called the initial matches for every frame.

\subsection{Dense Matching}

The iterative dense matching step uses the initial matches to obtain a complete matching between two frames. It starts 


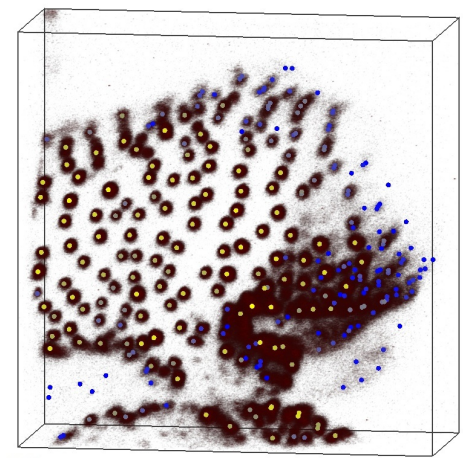

(a) Image 1

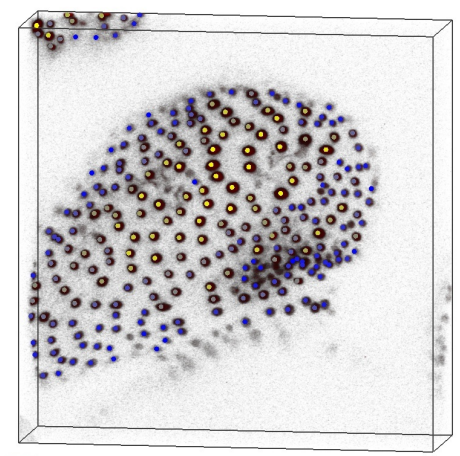

(b) Image 7

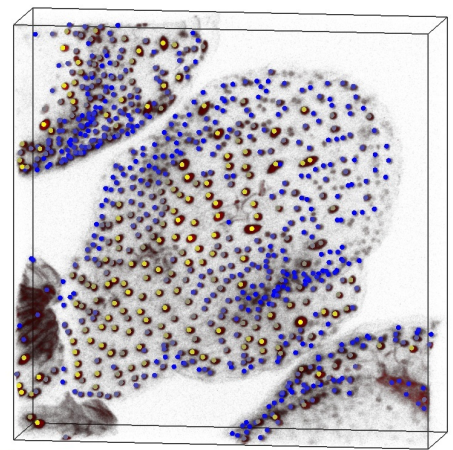

(c) Image 13

Fig. 1: The first, the seventh and the last image of the 3D CLSM data set rendered in BioView3D. The sepal changes significantly over the duration of the data set. The dots projected over the image volumes represent the detected centroids. Each centroid is assigned a confidence value between 0 and 1 visually color-coded from blue to yellow.

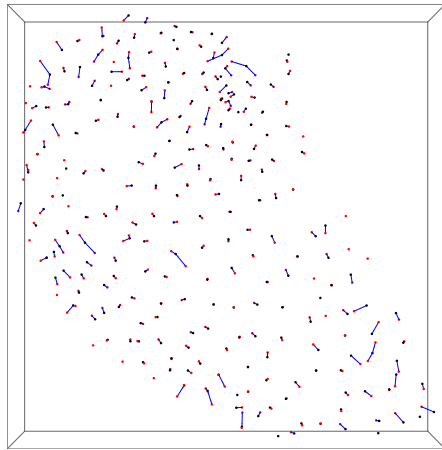

Fig. 2: Images 6 (black) and 7 (red) with their centroid matches displayed as blue lines. A 3D affine transformation is used to place both images in the same volume.

by selecting a centroid subset for every matched centroid in a frame as in Equation (1) and selecting its basis triplet as the centroid itself and its two nearest matched centroids. The matched centroids in the following frame are then used to describe a corresponding subset, of which we can assume the hash table it forms describes the same part of the sepal in two frames.

After all hash tables have been formed, matches between corresponding hash tables are found as in Equation (4). All hash table matches are then indexed according to their corresponding centroid matches and joined in one group. Initially only one-to-one matching is accepted. The resulting list of centroid matches is used for the next dense matching iteration until the set of matches no longer changes. Finally, one last iteration is performed that allows one-to-two matching, to account for cell division. The result can be seen in Figure 2 . The fact that this method reinforces correct matches based on local geometry also means that incorrect matches from a previous iteration are likely to be corrected.

\subsection{Image Registration}

The last step in our method is to register all images to make visual inspection of the data possible. First the centroid matches between images are connected to generate a lifeline that describes the behaviour of every matched nucleus, i.e. when it divided and where its daughter cells go.

To preserve as much information as possible, all images are transformed to the highest resolution available in the data set, but registered to the last frame, as it encompasses the biggest area. To keep the registration as natural as possible a transformation that, aside from the resolution scaling, only allows for rotation and translation is chosen. The rotation is centered around the lifeline of a central nucleus in the last frame to avoid a 'jittery' visualization over time. When the chosen lifeline consists of multiple daughter cells, the averaged location of all daughter cells is used as the anchor point $a$. All images then make the anchor point its origin, and the resolution scaling is applied. A rotation is computed for every frame from last to first using singular value decomposition (SVD). Formally, starting from the before last frame $N-1$, for $i=N-1 \ldots 1$

$$
\begin{aligned}
& M=\sum_{j=1}^{n}\left(m_{i}^{j}-a_{i}\right) R_{i+1}\left(m_{i+1}^{j}-a_{i+1}\right)^{\mathrm{T}} \\
& (U, S, V)=S V D(M) \\
& R_{i}=V U^{\mathrm{T}}
\end{aligned}
$$

Where $M$ is an accumulation matrix, $n$ is the number of matches between images $N_{i}$ and $N_{i+1}, m_{i}^{j}$ is the position of match $j$ in frame $i, U, S$ and $V$ are a $3 \times 3$ rotation and scaling matrices, and $R$ is the rotation used for the registration. No rotation is applied to frame $N$. The complete transformation for every CLSM volume then consists of rotation $R$, the anchor point translation and the voxel scaling. 


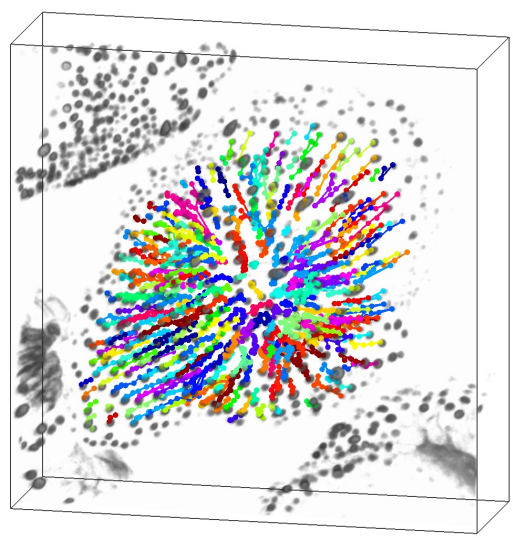

(a) All lifelines projected to image 13 .

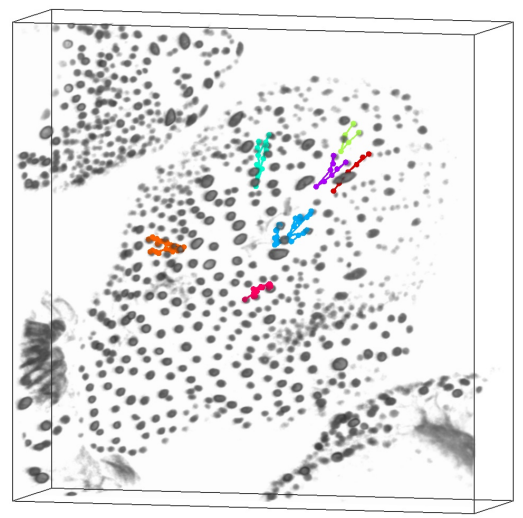

(b) Selected branching lifelines.

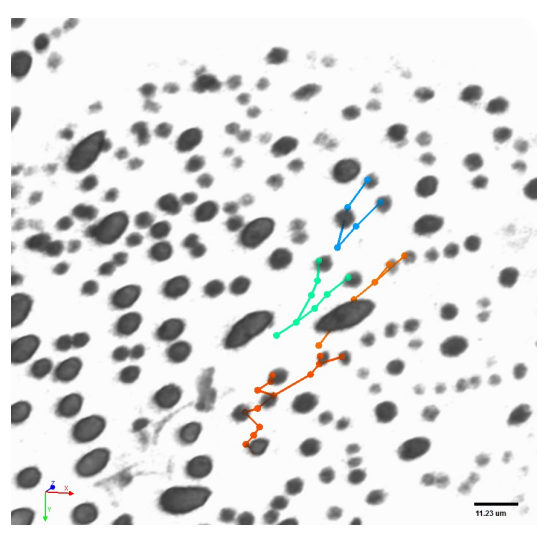

(c) Magnified lifelines.

Fig. 3: The final volume with overlayed lifelines projected to the last time frame. (a) All temporal lifeline projections. It can be seen that all projections are moving outward. (b) Some representative lifelines. Divisions can be seen as the projection branching outward. (c) Magnification of some lifelines.

After the transformation, the lifelines for all nuclei can then be projected in the volumes in different colors. Figure 3 shows three images with a few selected lifelines projected on the volume of the last image, all projected lifelines and a few lifelines zoomed in. It clearly shows that nuclei move outward and many divisions take place.

\section{DISCUSSION}

In this paper we present a novel cell matching and image registration algorithm, for the purpose of automating the cell tracking process in 3D CLSM microscopy images of the sepal of Arabidopsis thaliana. The cell matching algorithm consists of two steps: Initial matching and dense matching. The dense matching step iteratively matches all centroids between consecutive frames using the initial matches as the first iteration. The dense matching algorithm is able to correct most mismatches assuming that the initial matches are mostly correct, though this depends on the similarity of local geometry between two consecutive frames. This also determines the outcome of image registration, as it uses a lifeline based anchor point, which is subjective to the matching quality. The final registered 4D image along with cell lifelines for the described data set is accessible via web-based Bisque system [8] and can be downloaded and volume rendered in bioView3D [9]. Additional movies of the 3D rendering of the final result are also available on our website http://vision. ece. ucsb.edu/ fedorov/arabidopsis/.The algorithm could still be improved with an additional refinement step to correct wrongly matched centroids using a global cost function. We are working on extensive validation with new data sets and manually provided ground truth.

\section{Acknowledgments}

This research was partially sponsored by the NSF awards III0808772, \#0941717 and iPlant Collaborative \#DBI-0735191.

\section{REFERENCES}

[1] Adrienne H. K. Roeder, Vijay Chickarmane, Alexandre Cunha, Boguslaw Obara, B. S. Manjunath, and Elliot M. Meyerowitz, "Variability in the control of cell division underlies sepal epidermal patterning in Arabidopsis thaliana," PLoS Biol, vol. 8, no. 5, pp. e1000367, 052010.

[2] A. Roeder, A. Cunha, C. K. Ohno, and E. M. Meyerowitz, "Cell cycle regulates cell type in the Arabidopsis sepal," Development, vol. 139, pp. 4416-4427, 2012.

[3] Jim Swoger, " $4 \mathrm{~d}$ retrospective lineage tracing using spim for zebrafish organogenesis studies," Journal of Biophotonics, vol. 4, no. 1-2, 102010.

[4] Zhirong Bao, "Automated cell lineage tracing in Caenorhabditis elegans," Proceedings of the National Academy of Sciences of the United States of America, vol. 103, no. 8, pp. 2707-2712, 02 2006.

[5] Diego Piedrahita and Israel Hernández et al., "Silencing of cdk5 reduces neurofibrillary tangles in transgenic alzheimer's mice," Journal of Neuroscience, vol. 30, no. 42, pp. 1396613976, 2010.

[6] "Mathematica," http://www.wolfram.com/, Wolfram Mathematica.

[7] Haim J. Wolfson and Isidore Rigoutsos, "Geometric hashing: An overview," IEEE Comput. Sci. Eng., vol. 4, no. 4, pp. 10-21, Oct 1997.

[8] "Resulting 4d image," http://bisque.ece.ucsb. edu/client_service/view?resource=http: //bisque.ece.ucsb.edu/data_service/image/ 2592269, Resulting 4D image and lifelines accessible through the Bisque system.

[9] "bioview3d," http://www.bioimage.ucsb.edu/ downloads/BioView3D, Biological 3D/4D image renderer by the Center for Bio-Image Informatcs. 